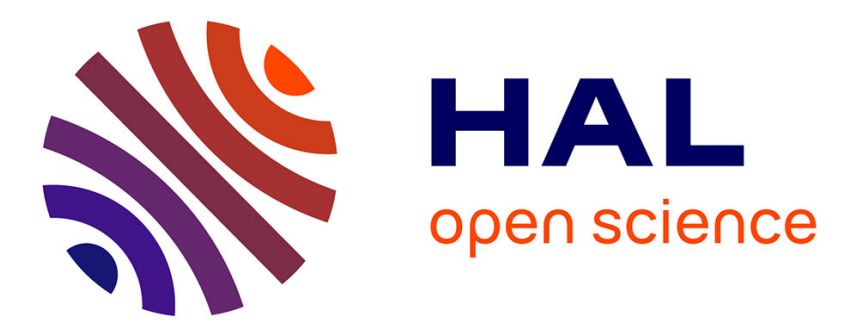

\title{
Actualisme, antiprogressionnisme, catastrophisme et créationnisme dans l'oeuvre d'Alcide d'Orbigny
}

\author{
Jean Gaudant
}

\section{To cite this version:}

Jean Gaudant. Actualisme, antiprogressionnisme, catastrophisme et créationnisme dans l'oeuvre d'Alcide d'Orbigny: Suivi de: "Sur deux aspects de l'oeuvre d'Alcide d'Orbigny" (Michel Rioult) et "Observations relatives au débat sur actualisme et catastrophisme chez Alcide d'Orbigny" (A. Fediaevsky, G. Gohau, G. Laurent). Travaux du Comité français d'Histoire de la Géologie, 1982, 1ère série, pp.66-75. hal-00951385v2

\section{HAL Id: hal-00951385 \\ https://hal.science/hal-00951385v2}

Submitted on 25 Feb 2014

HAL is a multi-disciplinary open access archive for the deposit and dissemination of scientific research documents, whether they are published or not. The documents may come from teaching and research institutions in France or abroad, or from public or private research centers.
L'archive ouverte pluridisciplinaire HAL, est destinée au dépôt et à la diffusion de documents scientifiques de niveau recherche, publiés ou non, émanant des établissements d'enseignement et de recherche français ou étrangers, des laboratoires publics ou privés. 


\title{
TRAVAUX \\ DU \\ COMITÉ FRANÇAIS D'HISTOIRE DE LA GÉOLOGIE \\ - Première série - \\ (1982)
}

\author{
J ean GAUDANT \\ ACTUALISME, ANTIPROGRESSIONNISME, \\ CATASTROPHISME ET CREATIONNISME DANS \\ L'OEUVRE D'ALCIDE D'ORBIGNY (1802 - 1857)
}

\begin{abstract}
COMITÉ FRANÇAIS D'HISTOIRE DE LA GÉOLOGIE (COFRHIGEO) (Séance du 24 mars 1982)
"Je n'ai jamais eu pour but, dans mes travaux, que l'avancement de la science à laquelle j'ai consacré mon existence" (A. d'Orbigny, 1855)

Bien qu'il se soit agi d'un ancien président de la Société géologique de France, à laquelle il avait dédié la "Paléontologie française", il fallut attendre une vingtaine d'années pour que cette société publie enfin la notice biographique d'Alcide d'Orbigny. P. Fischer y rappelait: "Plus de vingt ans se sont écoulés depuis la mort d'Alcide d'Orbigny. Les luttes ardentes soulevées par l'apparition de ses doctrines se sont éteintes; les idées justes, pratiques, qu'il a produites, ont été acceptées; en un mot, le jugement impartial de la postérité commence pour ses oeuvres" (1). En effet, de son vivant, l'oeuvre de Systématicien et de Biogéographe d'Alcide d'Orbigny souleva d'acerbes critiques de la part des Zoologistes, tandis que ses conceptions stratigraphiques étaient violemment contestées par la plupart des Géologues.
\end{abstract}

C'est dans son "Cours élémentaire de Paléontologie et de Géologie stratigraphiques" (1849 - 1852) qu'Alcide d'Orbigny exposa avec le plus de clarté ses idées sur l'histoire de "l'animalisation du globe". Nous ne reviendrons pas ici sur le rôle fondamental joué par A. d'Orbigny lors de la création d'une Stratigraphie véritablement scientifique car $M$. Rioult en a donné il y a quelques années une analyse très documentée (2). Nous insisterons en revanche sur les principaux aspects de sa philosophie naturelle.

\section{I - UNE METHODE ACTUALISTE}

Depuis les années 1830, deux conceptions radicalement différentes étaient préconisées d'une part en Angleterre, par Charles Lyell, d'autre part en France, par Constant Prévost qui, moins dogmatique que son célèbre confrère, concevait simplement l'Actualisme comme un moyen d'"éclairer l'histoire du passé par l'étude des phénomènes qui ont lieu sous nos yeux" (3).

Par certains côtés, l'Actualisme d'Alcide d'Orbigny n'est pas très éloigné de celui de Constant Prévost puisque son objectif est "d'arriver, par la connaissance des faits actuels, bien constatés, à reconnaître ce qui s'est passé aux différentes époques géologiques" (4). L'auteur accorde la priorité à l'étude des animaux marins car ceux-ci "ont joué un bien plus grand rôle (que les êtres terrestres) aux époques passées". II considère en effet que c'est "d'après leur répartition sur les côtes, au sein des mers, d'après leurs limites d'habitation en latitude, d'après les lois qui président à leur distribution géographique ... qu'on pourra, par des comparaisons scrupuleuses, dire avec quelque certitude quelles ont été les circonstances d'existence des êtres éteints" (5).

Toutefois, la Paléontologie implique non seulement une connaissance des "conditions d'existence" mais aussi des "conditions dans lesquelles les êtres sont détruits, naturellement ou par des événements fortuits, comme les inondations pour les espèces terrestres, les coups de vent, les tremblements de terre pour les espèces marines ou fluviales" (6). Enfin, il importe d'étudier les "divers modes de décomposition auxquels ces corps, privés de vie, sont soumis" (7). 
L'emploi de la méthode actualiste a permis à A. d'Orbigny d'acquérir "la conviction que des circonstances analogues (à ce qui se passe maintenant dans la nature) ont dû présider (au dépôt des couches terrestres) et ont donné, dans les mêmes conditions, des résultats identiques" (8). Notre auteur considère enfin que "l'étude de la distribution géographique des animaux est d'une extrême importance en Paléontologie, puisque, procédant du connu à l'inconnu, elle est destinée à nous révéler, par les lois qui président, aujourd'hui, à la distribution géographique et isotherme des êtres vivants, ce qui s'est manifesté aux diverses époques de l'animalisation de notre globe" (9). II serait en effet possible, grâce aux connaissances acquises de cette manière, de déterminer "les conditions d'existence des espèces perdues".

Néanmoins, dans le second volume de son "cours élémentaire", Alcide d'Orbigny admettait une exception au principe actualiste. Dans un cas, il lui fallait reconnaître que le "fil des opérations est rompu", pour reprendre la formule de Cuvier. En effet, une modification climatique radicale se serait produite lors de la "dernière création contemporaine de l'homme" car "les lignes isothermes si tranchées, qui cantonnent aujourd'hui, sur la terre, les êtres par zones de température, n'auraient ... commencé à se tracer qu'avec la faune actuelle" (10).

\section{II - L'HOSTILITE AU PROGRESSIONNISME}

Parmi les idées qui étaient discutées vers la fin de la première moitié du XIXè siècle, "celle qui se rapporte à la marche successive de l'animalisation sur le globe, depuis les temps géologiques les plus reculés jusqu'à l'époque actuelle" était pour A. d'Orbigny "l'une des questions les plus importantes de la zoologie générale" (11). Quelques années auparavant, cette question avait été débattue par W. Buckland (1836) dont l'ouvrage intitulé "La Géologie et la Minéralogie dans leurs rapports avec la Théologie naturelle" avait été traduit en français en 1838. Bien que diluvianiste - il avait du Déluge une conception beaucoup plus radicale que Cuvier - W. Buckland estimait que "les formes animales les plus perfectionnées deviennent graduellement de plus en plus abondantes à mesure que nous avançons des séries de dépôt les plus anciennes vers les plus récentes" (12). ॥I était approuvé en cela par A. Sedgwick (1850) qui considérait que "l'élévation de la faune des périodes successives n'a pas été faite par transmutation mais par additions créatrices" (13).

Alcide d'Orbigny s'opposa violemment à ces conceptions. Après avoir constaté que "les ordres d'animaux sont d'autant plus nombreux qu'ils se rapprochent davantage de notre époque; qu'ils sont, en un mot, dans une progression croissante de nombre de terrains les plus anciens aux plus modernes, et qu'aujourd'hui les ordres d'animaux sont à leur maximum numérique de développement" (14), il précisait son analyse en comparant époque après époque le nombre de genres appartenant à chaque Ordre. Cela le conduisait à conclure: "quand on voit 13 ordres sur 77, ou plus du sixième de l'ensemble numérique, se trouver dans la période décroissante de développement de formes zoologiques, on doit naturellement en conclure que toutes les séries animales n'ont pas suivi une marche uniforme dans les âges du monde. On y voit encore une exception importante à cette loi trop généralement admise du perfectionnement progressif des êtres, en marchant des époques anciennes aux plus modernes" (15). Un examen plus approfondi lui permettait en outre de souligner que, parmi les 13 ordres cités précédemment, "6 ou près de la moitié de l'ensemble, entrent dans la période décroissante avec la première époque de l'animalisation du globe" (c'est-à-dire au Paléozoïque) (16). Comme le fait remarquer A. d'Orbigny, "ce résultat est tout à fait contraire au perfectionnement progressif puisque la moitié de l'ensemble commence sur le globe par leur maximum de développement de formes zoologiques, et s'est, au contraire, toujours trouvée dans la période décroissante jusqu'à notre époque" (17). A l'appui de son argumentation, l'auteur pouvait encore ajouter le fait que, parmi les Mammifères, deux ordres: les Pachydermes et les Edentés, sont actuellement dans leur période décroissante alors que, "si l'hypothèse du perfectionnement progressif existait, on devrait trouver tous les ordres dans la période décroissante parmi les animaux rayonnés les plus imparfaits, et aucun parmi les animaux vertébrés les plus parfaits" (18).

Comme, en réalité, les proportions diffèrent peu d'un embranchement à l'autre, l'auteur est conduit à proposer une interprétation selon laquelle les quatre grands embranchements (Rayonnes, Mollusques, Annelés et Vertébrés) ont "marché ... sur quatre lignes parallèles indépendantes".Cette interprétation serait confirmée par le fait que ces quatre grands embranchements étaient déjà représentés au Paléozoïque, "ce qui prouverait que tous sont nés avec la première grande époque du monde animé" (19). A. d'Orbigny prétendait même que, sur les quatre classes de Vertébrés - les Amphibiens ou Batraciens étaient alors considérés comme des Reptiles -, "depuis la première période géologique jusqu'à présent, il y en a 2, les Reptiles et les Poissons, qui, au lieu de marcher dans ces âges des plus imparfaits aux plus parfaits, ont montré les plus parfaits les premiers, les moins parfaits dans les derniers, en suivant une marche entièrement opposée au perfectionnement successif des organes" (20). 
Passant ensuite à des arguments physiologiques, l'auteur faisait également remarquer que "si le perfectionnement progressif existait, on devrait trouver tous les animaux sans organe spécial de respiration, avec les premiers âges du monde, et les autres devraient paraître successivement, suivant leur degré de perfection; mais au contraire, tous les modes différents de respiration arrivant à la fois sur la terre, on en doit conclure que ce perfectionnement progressif n'existe pas" (21).

\section{III - UN CATASTROPHISME A REPETITION}

La discontinuité des faunes entre les étages successifs est l'idée cardinale sur laquelle Alcide d'Orbigny a établi sa Stratigraphie. II l'explique ainsi:

"Quand, sur les lieux, dans les étages qui se sont succédé régulièrement, on cherche le mode de distribution de ces faunes successives, on trouve toujours (à peu d'exceptions près) que dans les dernières couches de l'étage inférieur s'arrête la faune de cet étage; que là, elle s'est complètement anéantie: car les premières couches fossilifères de l'étage qui le recouvre renferment, de suite, des êtres très-différents des premiers et constituant une faune distincte de la faune de l'autre étage. Il résulte de ces faits, que tout le monde peut constater dans la nature et à toutes les époques géologiques, que chacun des étages qui se sont succédé dans les âges du monde renferme sa faune spéciale, bien tranchée, distincte des faunes inférieures et supérieures, et que ces faunes ne se sont pas succédé par passage de forme, ou par remplacement graduel, mais bien par anéantissement brusque" (22).

Suivant en cela Léonce Elie de Beaumont (23), qui interprétait les mouvements de l'écorce terrestre comme les conséquences de la contraction de la Terre, produite par son refroidissement progressif, A. d'Orbigny établissait une relation directe entre ces perturbations et les limites d'étages géologiques. II croyait ainsi "pouvoir attribuer aux seules perturbations géologiques, l'anéantissement complet des races d'animaux terrestres, gui couvraient le globe aux dernières époques antérieures à la nôtre,et leur dépôt dans les limons rougeâtres, à tous les niveaux terrestres, depuis le bord de la mer jusqu'à 4000 mètres au-dessus, sur les plateaux des Andes" (24). En généralisant, il était conduit à admettre que, "chaque fois qu'un système de montagnes a surgi au-dessus des océans, la faune existante a été anéantie par le mouvement prolongé des eaux, sur les points disloqués, et même sur ceux qui ne le sont pas; et une nouvelle période d'existence ne s'est manifestée que longtemps après le repos de la nature " (25). Comme A. d'Orbigny admettait que l'histoire de la Vie pouvait se décomposer en 28 étages (y compris la période contemporaine), il lui fallait donc supposer que, 27 fois, les faunes dont les restes sont conservés dans les terrains sédimentaires, ont été anéanties. Comme la cause de ces anéantissements ne peut être recherchée dans une modification profonde des conditions d'existence, il ne reste plus, pour expliquer ce fait "que les causes puissantes ..., c'est-à-dire l'effet des perturbations géologiques sur les faunes terrestres et marines" (25). Le recours à des perturbations géologiques universelles permettait même de justifier l'anéantissement global des faunes. Pour A. d'Orbigny, le Déluge pourrait être la conséquence de la dernière de ces perturbations géologiques et il le considère comme "le résultat d'oscillations considérables du sol, opérées depuis la création de l'homme, qui ont mis les eaux en mouvement sur une si vaste échelle" (27).

Enfin, la difficulté suscitée par l'existence - exceptionnelle à ses yeux -d'espèces "identiques" dans deux étages consécutifs pourrait être résolue en admettant que ces espèces ne sont connues que dans "quelques étages séparés sans doute par des perturbations géologiques de moindre valeur que ceux où aucune espèce commune ne s'est montrée jusqu'à présent" (28).

\section{IV - DES CREATIONS SUCCESSIVES}

Dès 1849, Alcide d'Orbigny avait souligné le fait qu'on ne peut observer aucune transition entre les animaux qui vivaient au cours d'époques successives, ce qui le conduisit à admettre que ces animaux se sont succédé "non par passage, mais par extinction des races existantes et par création successive des espèces à chaque époque géologique" (29). Trois ans plus tard, il admettait que "rien ne peut nous dévoiler le mystère qui se rattache aux créations successives de la première jusqu'à la dernière époque du monde animé. Nous voyons en effet apparaître simultanément sur tous les points du globe à la fois une multitude d'êtres différents, appartenant à tous les grands types d'animaux, sans que rien les annonce sur la terre, sans que rien les sépare des époques inanimées antérieures" (30).

Aussi pouvait-il retracer de la façon suivante l'histoire de la Vie sur la Terre: "Une première création s'est montrée avec l'étage silurien. Apres l'anéantissement de celle-ci, par une cause géologique quelconque, après 
un laps de temps considérable, une seconde création a eu lieu dans l'étage devonien; et successivement vingt sept fois des créations distinctes sont venues repeupler toute la terre de ses plantes et de ses animaux, à la suite de chaque perturbation géologique qui avait tout détruit dans la nature vivante" (31).

\section{V - CONCLUSION}

II peut paraître surprenant de constater que la pensée d'Alcide d'Orbigny réunissait des composantes actualiste et catastrophiste que l'on peut juger inconciliables en première analyse. En cela,A. d'Orbigny doit être considéré comme une figure marquante de ce que R. Hooykaas a appelé le "catastrophisme actualiste" (32) dont L. Elie de Beaumont fut le principal théoricien. Toutefois, Alcide d'Orbigny fut beaucoup plus que cela, au point qu'en dépit d'une véritable conspiration du silence, dont son successeur dans la chaire de Paléontologie du Muséum national d'Histoire naturelle fut le premier artisan (33), il mérite pleinement d'être considéré comme l'un des géants de l'histoire de la Paléontologie. Et même si son nom semble encore ignoré par M.J.S. Rudwick (34), on peut affirmer, sans risque d'être contredit, qu'au moins par sa dimension stratigraphique, l'oeuvre monumentale d'Alcide d'Orbigny - elle excède 10000 pages imprimées! - fut de celles qui contribuèrent à donner une "signification aux fossiles".

\section{References et bibliographie}

(1) P. Fischer (1878), Bull. soc. géol. France, (3), 6, p. 434 - 453.

(2) M. Rioult (1971), Mém. Bur. Rech. géol. min., 75, p. 17 - 33.

(3) Article "Terrain" in Dictionnaire classique d'Histoire naturelle, 16, p. 133 - 175 (1830).

(4) Cours élémentaire de Paléontologie et de Géologie stratigraphiques, 1, p.6 (1849).

(5) Ibid., p. 6.

(6) Ibid., p. 6.

(7) Ibid., p. 6.

(8) Ibid., p. 71.

(9) ibid., p. 92.

(10) ibid., 2 (1), p. 242 (1852).

(11) ibid., p. 217.

(12) La Géologie et la Minéralogie dans leurs rapports avec la Théologie naturelle, 1, p. 101 (1838).

(13) A discourse on the Studies of the University, Preface (1850).

(14) Cours élémentaire..., 2 (1), p. 220 (1852).

(15) ibid., p. 220 - 221.

(16) ibid., p. 221.

(17) ibid., p. 221.

(18) ibid., p. 224.

(19) ibid., p. 224-225.

(20) ibid., p. 230. A. d'Orbigny considérait que les Poissons cycloïdes et cténoïdes étaient moins parfaits que les Poissons du Paléozoïque. De même, parmi les Reptiles, les Ophidiens et les Batraciens seraient "les derniers en perfection". 
(21) ibid., p. 238.

(22) ibid., p. 252.

(23) Ann. Sci. natur., 18, p. 5 - 25 et 284 - 416 (1829); ibid., 19, p. 5 -99 et 177 - 240 (1830).

(24) Cours élémentaire ..., 1, p. 130 (1849).

(25) ibid., p. 135.

(26) ibid., 2 (1), p. 253 (1852).

(27) ibid., 2 (2), p. 835 (1852).

(28) ibid., 2 (1), p. 256 (1852).

(29) Ibid., 1, p. 156 (1849).

(30) Ibid., 2 (1), p. 251 (1852).

(31) Ibid., 2 (1), p. 251.

(32) Continuité et discontinuité en géologie et biologie, p. 22 (1970).

(33) A. d'Archiac ne mentionne même pas le nom d'Alcide d'Orbigny dans son "Précis de l'histoire de la Paléontologie stratigraphique" publié dans le "Cours de Paléontologie stratigraphique" (1862).

(34) The Meaning of Fossils, rev. ed. (1976).

\section{Michel RIOULT Sur deux aspects de l'oeuvre d'Alcide d'Orbigny}

Après les exposés très documentés de Madame F. Legré-Zaidline et de M. J. Gaudant, je reviendrai sur deux aspects de l'oeuvre d'A.d'Orbigny pour en souligner l'originalité et l'interdépendance : la classification stratigraphique et l'utilisation des hypothèses sur les révolutions du globe et sur les créations successives, telles qu'elles sont présentées par A. d'Orbigny, cinq ans seulement avant sa mort, dans son "cours élémentaire de Paléontologie et de Géologie stratigraphiques".

\section{Classific ation stratigraphique d'A. d'Orbigny}

Cette classification s'est précisée entre 1840 (Paléontologie française) et 1852 (Cours élémentaire..). Elle arrive alors après celles qui furent successivement proposées par Alexandre Brongniart, de la Bêche, Buckland et d'Omalius d'Halloy, entre autres. Mais les différentes analyses critiques de l'oeuvre d'A. d'Orbigny ont trop souvent laissé dans l'ombre les qualités pour mieux s'attaquer aux travers d'un ensemble de propositions ambitieuses. La longue démarche du stratigraphe repose en fait sur une masse considérable de connaissances acquises sur le terrain, aussi bien sur les côtes que dans les bassins sédimentaires de France ou d'Amérique du Sud, sur une imposante littérature géologique, paléontologique, zoologique et botanique qu'il s'est astreint à dépouiller, sur une énorme quantité de fossiles qu'il a étudiée dans les collections nationales, régionales ou dans ses récoltes personnelles, et enfin sur l'important volume de courrier qu'il a échangé avec son réseau de correspondants français et étrangers.

Après Alexandre de Humboldt - qui l'a parrainé dans son grand voyage en Amérique méridionale - il fut un des tout premiers naturalistes à pouvoir directement comparer ses observations géologiques de part et d'autre de l'Atlantique. Cette expérience d'une vie est à la base d'une largeur de vue peu commune et impose, en ce qui le concerne, un certain recul pour juger ses conclusions. Ses contemporains européens tentaient d'établir des corrélations au sein d'un même bassin ou dans des bassins adjacents, sur des bases uniquement 
paléontologiques. A. d'Orbigny proposait des unités de mesure du temps géologique beaucoup plus larges, testées à travers plusieurs bassins sédimentaires et partiellement sur deux continents, fondées simultanément sur les caractères lithologiques et paléontologiques, ainsi que sur les relations géométriques des formations.

En recherchant des critères nouveaux pour délimiter et définir ses "étages", il tentait de se dégager des obstacles linguistiques et d'échapper aux querelles d'écoles et aux contingences faciologiques régionales. Avec sa classification stratigraphique, A. d'Orbigny proposait un langage commun à tous les géologues, indépendant du chaos naissant de la lithostratigraphie, et dans le même temps, un système de conventions scientifiques internationales référenciées à des séries locales ou régionales. Cette classification devait jouer pour les Sciences de la Terre le même rêle que les nomenclatures raisonnées, ordonnées et pratiques de Linné pour les Sciences de la Vie ou de Berzélius en Chimie. Ce véritable OUTIL de TRAVAIL permettait d'émerger des inventaires régionaux et d'aborder la paléogéographie sur de meilleures bases.

\section{Utilisation par d'Orbigny de I'hypothèse des créations successives et de celle des révolutions du globe}

La liaison étroite entre l'histoire de la Vie et celle de la Terre imposait la recherche d'un fil directeur commun. D'un côté, le créationnisme traditionnel dérivait lentement depuis les travaux de Buffon et les tenants du dogme tentaient de le revigorer. Cuvier, en proposant les révolutions du globe, volait au secours des créations successives et des époques de la nature. L'uniformitarisme suggérait plutôt une continuité des conditions physiques et chimiques à la surface de la terre, jalonnée par des variations d'intensité des différents paramètres.

"La nature avait au commencement de chaque époque des forces créatrices qui n'agissent pas maintenant" : A. d'Orbigny reprenait à son compte cette idée émise en 1828 par l'Abbé Croizet. En effet, il constatait un renouvellement des faunes à la base de chacun de ses étages : cette hypothèse s'accordait aussi avec sa distinction entre les "causes passives permanentes" et les "causes géologiques accidentelles". Les créations successives correspondaient à ses "faunes spéciales" et il les préférait à une création continue telle que la présentait Lamarck, car il en rejetait une notion de progrès, trop finaliste et anthropocentrique à son gré. D'Orbigny ne reconnaît qu'un lent perfectionnement des espèces dans l'intervalle séparant deux bouleversements successifs, c'est-à-dire dans chacun de ses étages. Les révolutions du globe, fortement argumentées par Georges Cuvier qui se réfère aux Vertébrés, sont alors extrapolées aux Invertébrés par d'Orbigny qui peut difficilement démontrer l'anéantissement des peuplements marins à chaque révolution du globe, alors que chaque expédition scientifique d'alors rapporte des matériaux nouveaux et comble des lacunes dans la connaissance du monde animal ou végétal. D'Orbigny est bien fîxiste dans le cadre de la systématique en usage dans la première moitié $d u \mathrm{XIX}^{\circ}$ siècle, dont les genres et espèces ont une ampleur beaucoup plus grande qu'aujourd'hui. Ses espèces ont la valeur de genres ou de familles : dans ces limites, larges et floues, le lent perfectionnement estompe la portée des variations morphologiques observées, d'autant plus que la variabilité des Invertébrés se prête encore mal à l'étude en ce début de siècle.

L'expérience des milieux marins et continentaux actuels acquise par d'Orbigny sur le terrain, confère à son oeuvre géologique un cachet original, teinté d'un certain uniformitarisme en ce que les catastrophes invoquées sont toujours des paroxysmes de phénomènes naturels, de même nature que ceux dont il a pu constater les effets : tremblements de terre, éruptions volcaniques, raz de marées, inondations, sécheresses.. Mais ces bouleversements épisodiques seraient directement liés à la transformation de l'écorce terrestre et auraient des effets sur toute la surface du globe, avec des intensités différentes et des formes variées. Les périodes de sédimentation et de peuplement des terres et des mers sont contrôlées par les seules "forces passives incessantes" observées encore actuellement : les bouleversements des révolutions du globe sont soumis aux "forces géologiques fortuites"; accidentels, ils interrompent épisodiquement les premiers.

Dans son étude des séries anciennes, A. d'Orbigny recherche le plus petit DENOMINATEUR CHRONOLOGIQUE COMMUN : L'ETAGE. II a conclu que nulle part la sédimentation n'est un phénomène continu. Incontestablement, il est frappé par les discontinuités de sédimentation. II les utilise comme plansrepères ou dates dans les successions sédimentaires, au Jurassique et au Crétacé par exemple, et aussi bien dans ses milieux "côtiers" en bordure de massif ancien (Normandie, Poitou), que dans des milieux marins plus franchement ouverts, ses milieux "pélagiens" (Charente, Haute-Provence). D'Orbigny distingue dans ces "lignes de séparation des étages", trois types d'interruption de la sédimentation liés aux dislocations de l'écorce terrestre telles que les expliquait alors L. Elie de Beaumont. Les discordances de stratification, les surfaces de ravinement et les simples surfaces durcies et perforées seraient pour d'Orbigny autant d'échos aux perturbations tectoniques invoquées. II insiste d'ailleurs sur toutes les modifications qui accompagnent ces discontinuités: 
changements de composition, de couleur, de granulométrie, remaniements, lacunes, renouvellement des faunes, ... "Catastrophiste" il l'est ici par nécessité, pour justifier la netteté des "lignes de séparation" et les "caractères tranchés" qui opposent les faunes spéciales qui se succèdent. Finalement, la fameuse phrase énonçant la liste des vingt-sept créations successives séparées par autant de révolutions du globe, isolée de son contexte et de ses applications par la critique, a déprécié l'ensemble de l'oeuvre et les démarches originales d'A. d'Orbigny.

Rejeté par les zoologistes et de nombreux géologues, miné par les échecs, les deuils et les fièvres, en proie à de perpétuelles difficultés professionnelles et financières, il mourra sans répondre à ses détracteurs. Et même son beau-frère, A. Gaudry qui fournira à la théorie évolutionniste ses premiers exemples d'enchaînements de Vertébrés, se vit plus tard encore reproché sa ligne de pensée catastrophiste, héritée des longs échanges et de son amitié avec A. d'Orbigny. Les souvenirs laissés par les discussions entre Georges Cuvier et Geoffroy SaintHilaire, l'opposition aux idées de Lamarck et l'engouement pour les idées de Darwin ont contribué à la disgrâce d'A. d'Orbigny dans la communauté scientifique française.

II devra affronter seul cette lourde conspiration du silence, faite de jalousie, de mépris et d'inertie : A. d'Archiac ignorera même les travaux d'A. d'Orbigny dans son "Cours" et dans son "Introduction à l'étude de la Paléontologie stratigraphique"... alors qu'il professait au Muséum d'Histoire Naturelle dans la chaire créée par A. d'Orbigny ! II ne se trouvera personne pour prendre la défense du secrétaire de la Société Géologique de France.

Accueillie avec enthousiasme par le réseau de correspondants provinciaux d'A. d'Orbigny, sa classification stratigraphique fut trop souvent mal comprise (même dans les terrains jurassiques et crétacés pour lesquels manifestement elle était faite),surtout par les stratigraphes en mal de créer un nouvel étage. Par contre, elle fût contestée et malmenée par ceux qui étudiaient les couches paléozoïques et cénozoïques. Plus mal à l'aise dans ces terrains et ignorant de nombreux travaux de langue française et anglaise, A. d'Orbigny avait commis de nombreuses maladresses. Entre autres, celle de dédier un "étage" à son ami Murchison pour les terrains compris entre le Silurien et le Dévonien, et celle de rejeter la nomenclature proposée par Charles Lyell pour les terrains tertiaires, alors qu'elle avait été retenue par Buckland et de nombreux géologues à sa suite. Mais surtout, d'Orbigny avait introduit une grande disproportion de volume et de temps entre les "étages" paléozoïques, mésozoïques et cénozoïques. Sa démarche sera pourtant reconnue par les Congrès géologiques internationaux à la fin du $\mathrm{XIX}^{\circ}$ siècle.

L'exposé des principes de base contenus dans le Cours élémentaire de Géologie et Paléontologie stratigraphiques reflète à mes yeux une vision fondamentale de la stratigraphie et de la paléogéographie. La nouveauté et l'euphorie intellectuelle qui ont assuré le succès du Darwinisme ont dévalué l'apport de cette paléontologie stratigraphique dû à $A$. d'Orbigny. Ce qui prévalut dans son "bannissement" n'a pourtant pas cessé de préoccuper les géologues et les paléontologistes. Ne discutent-ils pas encore des "crises paléobiologiques", des "explosions", des rythmes ou des extinctions ? D'autre part, ces discontinuités de sédimentation sont-elles dépourvues d'intérêt quand les géologues marins ou pétroliers les traquent sur chacun de leurs sismogrammes ? Le temps reste bien un paramètre continu, mais son enregistrement sédimentaire et biologique est toujours discontinu dans l'espace. Repère et indices retenus pour les datations relatives et les corrélations stratigraphiques ont tous des limites paléogéographiques naturelles à l'échelle globale.

Le langage stratigraphique, la recherche de critères de corrélation sédimentologiques, paléontologiques et structuraux, les esquisses paléogéographiques d'A. d'Orbigny destinés à mettre en place la répartition des êtres vivants, des mers et des terres pendant chaque "étage", sont des apports qui restent essentiels pour la géologie.

\section{Observations relatives au débat sur actualisme et catastrophisme chez Alcide d'Orbigny.}

\section{Alexandre FEDIAEVSKY}

Le catastrophisme est-il seulement un concept géologique à cette époque ? Nous sommes en plein début du romantisme avec son goût artistique pour les ruines et les cataclysmes historiques. II est vrai que beaucoup de contemporains ont vécu la Révolution comme une apocalypse. C'est le cas par exemple du poète BLAKE. 


\section{Gabriel GOHAU}

Je veux rappeler qu'historiquement l'actualisme combat sur deux fronts. Contre le catastrophisme, d'abord, contre ce qu'on peut nommer l'évolutionnisme géologique, ensuite. C'est-à-dire que Charles Lyell est partisan, à la fois, de la continuité des phénomènes et de la stabilité du globe à travers le temps : le présent explique d'autant mieux le passé que l'évolution de la terre a été peu sensible.

C'est Whewell qui nomme "catastrophistes" les adversaires de Lyell, privilégiant le couple continuitédiscontinuité au détriment de l'opposition stabilité-évolution. Mais dès 1841, Conybeare rappelait que le véritable problème concernait l'alternative modèle "historique" - modèle "stationnariste" (historical model, steady-state model). Cf. Rudwick, "A critique of Uniformitarian Geology - A letter from W.D. Conybeare to Ch. Lyell, 1841". Proc.Am.Phil.Soc., CXI (1967), 272-287.

Or, si du premier point de vue, d'Orbigny est manifestement dans le camp catastrophiste, du second il est moins éloigné de Lyell que les "progressionnistes" (Cuvier (?), Buckland, Sedgwick, M. de Serres, etc.), puisqu'il nie la progression du monde vivant. Cf. Orbigny, Cours élém. paléontologie et géol.strati., 1849-1851, 11,225232 (il accepte, par exception, une gradation des ordres de Mammifères, ibid, I,190). A. d'Orbigny se rapproche donc sur ce thème de Lyell, qui niait que les Mammifères fussent d'apparition récente. Cf. Lyell, Principles..., $2^{\circ}$ ed., 1832-1833,I,166sq.

\section{Goulven LAURENT}

L'oeuvre d'Alcide d'Orbigny contient certes des passages où cet auteur envisage favorablement l'activité des "causes actuelles". En fait, à peu près tous les géologues du 19ème siècle s'entendent pour utiliser cet instrument d'explication du passé, ce qui est très concevable dans la mesure où il se situent dans le sillage de Newton. Cuvier aussi le fait tout naturellement, lui qui essaie de reconstruire les êtres fossiles à partir des lois qui gouvernent l'organisation des animaux actuels, et qui reconnaît, à la suite des Saussure, Pallas et Dolomieu, que "le premier pas à faire pour deviner le passé, c'étoit de bien constater le présent" (1). Les époques géologiques n'ont pas été, en effet, des époques de révolutions permanentes : il y a eu des périodes de repos et des périodes d'agitation. Pour les périodes calmes, les agents actuels sont tout à fait acceptables.

Mais on ne peut pas dire de ces auteurs — ni de Cuvier, ni d'Alcide d'Orbigny - qu'ils soient actualistes pour autant. Ce qui définit l'actualisme, en effet, c'est l'affirmation que les causes actuelles suffisent à tout expliquer des événements qui se sont passés autrefois. Or il est évident que, pour eux, les "catastrophes" ne peuvent être expliquées par les causes aujourd'hui connues.

Cuvier articule bien cette différence : "vraies ou non, peu importe" écrit-il, les causes actuelles ne sont pas suffisantes pour un géologue, "puisque aucune cause lente ne peut avoir produit des effets subits". Et c'est bien pour cette raison, assure-t-il, que l'on s'est trouvé devant la "nécessité" de chercher "des causes différentes de celles que nous voyons agir aujourd'hui" (2). Pour l'explication des phénomènes extraordinaires, il ne reste donc que le recours au mystère, ou à l'intervention directe d'une puissance extérieure.

On ne peut s'empêcher d'évoquer par contraste la position toute contraire de Lamarck. Pour lui, il n'y a pas eu de cataclysmes universels, car des espèces "analogues" ont franchi ces obstacles prétendument infranchissables. Puisqu'il n'y a pas eu de catastrophes, il n'y a pas non plus à faire appel à des causes extraordinaires : les causes actuelles suffisent à expliquer tous les événements du passé.

La problématique lamarckienne des espèces "analogues" devait rapidement ruiner la thèse cuviérienne catastrophiste -Alcide d'Orbigny étant un des derniers à soutenir le catastrophisme absolu à répétition (27 fois !). En effet, de nombreux géologues et paléontologistes français (les Invertébristes, en particulier) : de Férussac, Lamouroux, A. Boué, sans parler d'Etienne Geoffroy Saint-Hilaire, ni bien sûr de Constant Prévost (ni de Blainville, Delamétherie, Marcel de Serres, d'Archiac, etc...), suivent, sur ce point, la doctrine anti-catastrophiste de Lamarck, en utilisant sa méthode. Ce n'est donc pas du côté de Cuvier ni de ses disciples, qu'il faut chercher les "prophètes" de l'actualisme, mais bien du côté du fondateur du Transformisme.

\section{Notes}


(1) Journal de Physique, t.52, 1801, p.255

(2) Discours sur les Révolutions de la Surface du Globe, 1825, p.42-43

\section{Voir aussi sur ce site :}

- Alcide d'Orbigny : un talentueux dessinateur naturaliste révélé par le legs Bedel-d'Orbigny, par Françoise LEGRÉ-ZAIDLINE (1997).

- L'oeuvre paléontologique d'Alcide d'Orbigny en Amérique du Sud, par Françoise LEGRÉ-ZAIDLINE (1982). 